\title{
A Two-parameter Deformation of Supergroup GL(1/2)
}

\author{
Sultan ABACI ÇELIK ${ }^{1}$
}

ABSTRACT: A new super-Hopf algebra, denoted by , is obtained by using the standard method (the RTT-relation) with an $R$-matrix which is a solution of the quantum Yang-Baxter equation.

Keywords: Yang-Baxter equation, super-Hopf algebra, quantum supergroup.

\section{GL(1|2) Süper Grubunun Bir İki-parametreli Deformasyonu}

ÖZET: Kuantum Yang-Baxter denkleminin çözümü olan bir $R$-matrisi yardımıyla, standard RTT-bağıntıSı kullanılarak ile gösterilen yeni bir süper-Hopf cebiri elde edilmiştir.

Keywords: Yang-baxter equation, super-hopf cebiri, kuantum super grup.

Sultan ABACI ÇELIKK (0000-0003-3465-8209), Yildiz Technical University, Faculty of Arts and Sciences, Departmet of Mathematics, İstanbul, Turkey

Sorumlu yazar/Corresponding Author: Sultan ABACI ÇELIK, sultan@yildiz.edu.tr 


\section{INTRODUCTION}

Quantum groups (Drinfeld, 1986) have a rich mathematical structure (Klimyk and Schmüdgen, 1997), (Majid, 1995). The standard method to construct a new algebra from a solution of the quantum Yang-Baxter equation (Yang, 1967) was initiated by Faddeev et al. in 1990. With this method, we will introduce a new superalgebra related to a $\mathbb{Z}_{2}$ graded $R$-matrix with two-parameter. The RTT-relation for the quantum supergroups has the same form as in the (Faddeev et al. in 1990), but matrix tensor product contains a factor (-1), as additional to the (Kulish and Sklyanin, 1982) related to $\mathbb{Z}_{2}$-grading (Berezin, 1987).

The tensor product of two even matrices $U$ and $V$ has the signs

$$
(U \otimes V)_{i j, k l}=(-1)^{\tau(j)(\tau(\mathrm{i})+\tau(\mathrm{k}))} U_{i k} V_{j l}
$$

where $\tau\left(U_{i j}\right)=\tau(i)+\tau(j)$. Because of this description, a matrix in the form $I \otimes U$ has the same block-diagonal form as in the standard (no-grading) case while a matrix in the form $U \otimes I$ contains the factor (-1) for odd elements standing at odd rows of blocks. To give a little explanation, we consider the matrix $U=\left(\begin{array}{ll}a & \alpha \\ \gamma & b\end{array}\right)$ appearing as $T_{33}$ on page

7, line 5. Then the tensor product of the matrices $U$ and $I=\left(\delta_{i j}\right)$ has the signs

$$
(U \otimes I)_{i j, k l}=(-1)^{\tau(j)(\tau(i)+\tau(k))} U_{i k} \delta_{j l} \text { and }(I \otimes U)_{i j, k l}=(-1)^{\tau(j)(\tau(i)+\tau(k))} \delta_{i k} U_{j l}=\delta_{i k} U_{j l}
$$

where $\delta_{i j}$ denotes the kronecker delta. So, we have, for example

$$
(U \otimes I)_{11,21}=+U_{12} \delta_{11}=\alpha, \quad(\mathrm{U} \otimes I)_{12,22}=-U_{12} \delta_{22}=-\alpha,(\mathrm{U} \otimes I)_{22,12}=-U_{21} \delta_{22}=-\gamma, \text { etc }
$$

In this paper, we construct a two-parameter deformation of the supergroup $G L(1 \mid 2)$, denoted by $G L_{p, q}(1 \mid 2)$. 


\section{MATERIAL AND METHODS}

Let $a, b, c, d, e, \alpha, \beta, \gamma, \delta$ be generators of an algebra $A$, where the generators $a, b, c, d, e$ are of grade 0 and the generators $\alpha, \beta, \gamma, \delta$ are of grade 1 . Let $O(M(1 \mid 2))$ be defined as the polynomial algebra $k[a, b, c, d, e, \alpha, \beta, \gamma, \delta]$. It will be sometimes more convenient and more illustrative to write a point $(a, b, c, d, e, \alpha, \beta, \gamma, \delta)$ of $O(M(1 \mid 2))$ in the matrix form, as a supermatrix,

$$
T=\left(\begin{array}{lll}
a & \alpha & \beta \\
\gamma & b & c \\
\delta & d & e
\end{array}\right)=\left(t_{i j}\right)
$$

We consider the $R$-matrix

$$
R=\left(\begin{array}{ccccccccc}
1 & 0 & 0 & 0 & 0 & 0 & 0 & 0 & 0 \\
0 & q^{-1} & 0 & 0 & 0 & 0 & 0 & 0 & 0 \\
0 & 0 & q^{-1} p^{-1} & 0 & 0 & 0 & 1-p^{-1} & 0 & 0 \\
0 & 1-p^{-1} & 0 & q p^{-1} & 0 & 0 & 0 & 0 & 0 \\
0 & 0 & 0 & 0 & p^{-1} & 0 & 0 & 0 & 0 \\
0 & 0 & 0 & 0 & 0 & q p^{-1} & 0 & p^{-1}-1 & 0 \\
0 & 0 & 0 & 0 & 0 & 0 & q & 0 & 0 \\
0 & 0 & 0 & 0 & 0 & 0 & 0 & q^{-1} & 0 \\
0 & 0 & 0 & 0 & 0 & 0 & 0 & 0 & p^{-1}
\end{array}\right)
$$

where $p, q \in \mathbb{C}-\{0\}$. This matrix satisfies the graded Yang-Baxter equation $R_{12} R_{13} R_{23}=R_{23} R_{13} R_{12}$ where $R_{12}=R \otimes I_{3}$, etc with the $3 \times 3$ identity matrix $I_{3}$.

The matrix $\hat{R}$ satisfies the $\mathbb{Z}_{2}$-graded braid relation

$$
\hat{R}_{12} \hat{R}_{23} \hat{R}_{12}=\hat{R}_{23} \hat{R}_{12} \hat{R}_{23}
$$


and the $\mathbb{Z}_{2}$-graded Hecke condition

$$
\left(\hat{R}-I_{9}\right)\left(\hat{R}+p^{-1} I_{9}\right)=0 .
$$

The eigenvalues of $\hat{R}$ are 1 and $-p^{-1}$ and it can be written, as a sum of projectors, in the form

$$
\hat{R}=-p^{-1} P_{-}+P_{+}
$$

where

$$
P_{-}=\frac{-\hat{R}+I_{9}}{1+p^{-1}}, \quad P_{+}=\frac{\hat{R}+p^{-1} I_{9}}{1+p^{-1}}
$$

provided that $1+p^{-1} \neq 0$. The projectors obey $P_{i} P_{j}=\delta_{i j} P_{j}$ (no summation) and $P_{-}+P_{+}=I_{9}$.

\section{RESULTS AND DISCUSSION}

In this section, we get the $(p, q)$-commutation relations of the elements of the supermatrix $T$ given in (1) and show that the algebra $O\left(G L_{p, q}(1 \mid 2)\right)$ is a super-Hopf algebra.

Theorem 3.1. A $3 \times 3$-supermatrix $\mathrm{T}$ is a $\mathbb{Z}_{2}$-graded quantum matrix if and only if

$$
\hat{R} T_{1} T_{2}=T_{1} T_{2} \hat{R}
$$

where $T_{2}=I_{3} \otimes T, T_{1}=P T_{2} P$ and $\hat{R}=P R$ with the super permutation matrix $P$. As a result of (3), the elements of the supermatrix $T$ satisfy the relations 


$$
\begin{aligned}
& a b=b a+q\left(1-p^{-1}\right) \gamma \alpha, \quad a c=p^{-1} c a, \quad a d=p d a, \\
& a e=e a+q(1-p) \delta \beta, \quad b c=q c b, \quad b d=p q^{-1} d b, \\
& b e=e b+q^{-1}(p-1) d c, c d=p q^{-2} d c, c e=p q^{-1} e c, d e=q e d, \\
& a \alpha=p q^{-1} \alpha a, \quad a \beta=q^{-1} p^{-1} \beta a, \quad a \gamma=q \gamma a, \quad a \delta=q \delta a, \\
& b \alpha=p q^{-1} \alpha b, \quad b \beta=\beta b+q^{-1}(p-1) \alpha c, \quad b \gamma=q \gamma b, \\
& b \delta=\delta b+q(1-p) \gamma d, \quad c \alpha=p q \alpha c, \quad c \beta=p q \beta c, \quad c \gamma=q \gamma c, \\
& c \delta=p \delta c, \quad d \alpha=q^{-1} p^{-1} \alpha d, \quad d \beta=p^{-1} \beta d, \quad d \gamma=q^{2} p^{-1} \gamma d, \quad d \delta=q \delta d, \\
& e \alpha=q^{-2} \alpha e+q^{-1}\left(p^{-1}-1\right) \beta d, \quad e \beta=q^{-1} p^{-1} \beta e, \quad e \gamma=q^{2} \gamma e+q(1-p) \delta c, \\
& e \delta=q \delta e, \alpha \beta=-q p^{-1} \beta \alpha, \quad \alpha \gamma=-q^{2} p^{-1} \gamma \alpha, \alpha \delta=-q^{2} \delta \alpha+q(p-1) d a, \\
& \beta \gamma=-q^{2} \gamma \beta+q(1-p) a c, \quad \beta \delta=-p q^{2} \delta \beta, \gamma \delta=-q^{-1} \delta \gamma, \\
& \alpha^{2}=\beta^{2}=\gamma^{2}=\delta^{2}=0 .
\end{aligned}
$$

Proof. Results can be obtained by making direct calculations.

One can see that when $p=q$, these relations coincide with those of $G L_{p, q}(1 \mid 2)$ given in (Celik, 2016).

Definition 3.1. The superalgebra $O\left(\mathrm{M}_{p, q}(1 / 2)\right)$ is the quotient of the free algebra $k<a, b, c, d, e, \alpha, \beta, \gamma, \delta>$ by the two-sided ideal $J_{p, q}$ constituted by the relations in (4) of Theorem 3.1.

Let $A$ and $B$ be two superalgebras. Then their tensor product $A \otimes B$ is a superalgebra with respect to tensor product of $A$ and $B$. The product rule for tensor product of superalgebras is given in the following definition. We denote by $\tau(a)$ the grade of an element $a \in A$.

Definition 3.2. If $A$ is a superalgebra, then the product rule in the superalgebra $A \otimes A$ is described by 


$$
\left(a_{1} \otimes a_{2}\right)\left(a_{3} \otimes a_{4}\right)=(-1)^{\tau\left(a_{2}\right) \tau\left(a_{3}\right)} a_{1} a_{3} \otimes a_{2} a_{4}
$$

where $a_{i}$ 's are homogeneous elements in the superalgebra $A$.

The quantum superdeterminant for the supermatrix $T$ in the block form is given by (cf. Kobayashi and Uematsu, 1992)

$$
s \operatorname{det}(T)=\operatorname{det}\left(A-B D^{-1} C\right)(\operatorname{det}(D))^{-1}
$$

and it is not a central element. If the inverse of the quantum superdeterminant $s \operatorname{det}(T)$ exists, then the algebra $O\left(G L_{p, q}(1 \mid 2)\right)$ has a super-Hopf algebra structure. The superHopf algebra structure of $O\left(G L_{p, q}(1 \mid 2)\right)$ is given in below.

Theorem 3.2. The algebra $O\left(G L_{p, q}(1 \mid 2)\right)$ has a unique super-Hopf algebra structure with co-maps $\Delta, \varepsilon$ and $S$ such that

$$
\Delta\left(t_{i j}\right)=\sum_{k=1}^{3} t_{i k} \otimes t_{k j}, \varepsilon\left(t_{i j}\right)=\delta_{i j} \text { and } \mathrm{S}(T)=T^{-1} .
$$

Proof. The following properties of the co-structures can easily verified:

The comultiplication $\Delta$ is coassociative in the sense that

$$
(\Delta \otimes \mathrm{id}) \circ \Delta=(\mathrm{id} \otimes \Delta) \circ \Delta
$$

where id: $A \rightarrow A$ denotes the identity map and $\Delta(u v)=\Delta(u) \Delta(v), \Delta(1)=1 \otimes 1$.

The counit $\varepsilon$ has the property

$$
m \circ(\varepsilon \otimes \mathrm{id}) \circ \Delta=\mathrm{id}=m \circ(\mathrm{id} \otimes \varepsilon) \circ \Delta
$$

where $m: A \otimes A \rightarrow A$ and $\varepsilon(u v)=\varepsilon(u) \varepsilon(v), \quad \varepsilon(1)=1$. 
The coinverse $S$ satisfies

$$
m \circ(S \otimes \mathrm{id}) \circ \Delta=\varepsilon=m \circ(\mathrm{id} \otimes S) \circ \Delta
$$

and $S(u v)=(-1)^{\tau(u) \tau(v)} S(v) S(u), S(1)=1$.

Definition 3.3. The super-Hopf algebra $O\left(G L_{p, q}(1 \mid 2)\right)$ is called the coordinate algebra of the quantum supergroup $G L_{p, q}(1 \mid 2)$.

\section{A discussion of some submatrices}

Here are a few comments about some submatrices of $T$.

1. Let us first consider the even $2 \times 2$-submatrix $T_{33}=\left(\begin{array}{ll}a & \alpha \\ \gamma & b\end{array}\right)$ which forms subgroup $G L_{p, q}(1 / 1)$ with the commutation rules

$$
\begin{aligned}
& a \alpha=p q^{-1} \alpha a, \quad a \gamma=q \gamma a, \quad b \alpha=p q^{-1} \alpha b, \quad b \gamma=q \gamma b, \\
& a b=b a+q\left(1-p^{-1}\right) \gamma \alpha, \quad \alpha \gamma=-q^{2} p^{-1} \gamma \alpha, \quad \alpha^{2}=\gamma^{2}=0 .
\end{aligned}
$$

These relations coincide with relations in (Dabrowski and Wang, 1991) when $p$ is replaced by $p q$. If we assume that the formal inverse $b^{-1}$ of $b$ exists, then the quantum superdeterminant is given by the expression

$$
s \operatorname{det}\left(T_{33}\right)=a b^{-1}-\alpha b^{-1} \gamma b^{-1}
$$

and it is a central element of the quantum superalgebra $O\left(G L_{p, q}(1 \mid 1)\right)$.

It can be seen in a similar way that the even 2x2-submatrix $T_{22}=\left(\begin{array}{ll}a & \beta \\ \delta & e\end{array}\right)$ forms subgroup $G L_{p, q}(1 \mid 1)$ with the defining commutation relations. 
2. We now consider an algebra $A$ generated by the elements $a, \alpha, \delta, d$ and defining commutation rules

$$
\begin{aligned}
& a \beta=q^{-1} p^{-1} \beta a, \quad a \delta=q \delta a, \quad e \beta=q^{-1} p^{-1} \beta e, \quad e \delta=q \delta e, \\
& a e=e a+q(1-p) \delta \beta, \quad \beta \delta=-p q^{2} \delta \beta, \quad \beta^{2}=\delta^{2}=0 .
\end{aligned}
$$

Obviously these relations represent a two-parameter deformation of the algebra $A$. Here the generators $a$ and $d$ are almost even (bosonic) and the generators $\alpha$ and $\delta$ are almost odd (fermionic). Indeed, as $p, q \rightarrow 1$ the algebra $A$ with these relations becomes a superalgebra. However, submatrices of the form $T_{23}=\left(\begin{array}{ll}a & \alpha \\ \delta & d\end{array}\right)$ with the defined relations (except for $p=q=1$ ) do not form a subgroup $G L_{p, q}(1 \mid 1)$. It seems that such matrices are related to the super braided matrices (Majid, 1991). If so, this will be addressed in another study.

3. The $2 \times 2$-submatrix $T_{23}=\left(\begin{array}{ll}b & c \\ d & e\end{array}\right)$ forms subgroup $G L_{p, q}(2)$ subject to the relations

$$
\begin{aligned}
& b c=q c b, \quad b d=p q^{-1} d b, \quad c e=p q^{-1} e c, \quad d e=q e d, \\
& b e=e b+q^{-1}(p-1) d c, \quad c d=p q^{-2} d c .
\end{aligned}
$$

These relations coincide with relations given in (Schirrmacher et al., 1991) when $q$ is replaced by $p$ and $p q^{-1}$ is replaced by $q$. The quantum determinant is given by

$$
\operatorname{det}\left(T_{11}\right)=b e-q c d=e b-q^{-1} d c
$$

and it is not in the centre of the algebra $O\left(G L_{p, q}(2)\right)$, but it becomes central if $p=q^{2}$. 


\section{CONCLUSION}

An $R$-matrix satisfying quantum YangBaxter equation was found, and using this matrix, deformation of the supergroup with a two-parameter was obtained and it shown that

\section{REFERENCES}

Berezin F A, 1987. Introduction to Algebra and Analysis with Anticommuting Variables. Springer +Science Busines Media Dordrecht-Holland.

Celik S, 2016. Bicovariant Differential Calculus on the Quantum Superspace $\square_{q}(1 \mid 2)$. Journal of Algebra and Its Applications, 15:1650172-1-17.

Dabrowski L, and Wang L, 1991. Two-parameter Quantum Deformation of GL(111). Physics Letters B, 266:51-54.

Delta Function Interaction. Physical Review Letters, 19:13121314.

Drinfeld V G, 1986. Quantum groups, Procedings of the International Congress of Mathematicians No: 1, pp. 798820, Berkeley-USA.

Faddeev L D, Reshetikhin N Y and Takhtajan L A, 1990. Quantization of Lie groups and Lie algebras. Leningrad Mathematical Journal, 1:193-225.

Klimyk A and Schmüdgen K, 1997. Quantum Groups and Their Representations. Texts and Monographs in Physics, Springer, New York-USA. has a super-Hopf algebra structure, as usual

Acknowledgements: I would like to express my deep gratitude to the referees for many comments and suggestions on the manuscript.

Kobayashi T and Uematsu T, 1992. Differential Calculus on the Quantum Superspace and Deformation of Phase Space. Zeitschrift für Physik C Particles and Fields, 56:193-199.

Kulish P P and Sklyanin E K, 1982. Solutions of the YangBaxter Equation. Journal of Soviet

Mathematics, 19:1596-1620.

Majid S, 1991. Examples of Braided Groups and Braided Matrices. Journal of Mathematical Physics, 32:3246-325.

Majid S, 1995. Foundtions of Quantum Group Theory. Cambridge University press, England.

Schirrmacher A, Wess J and Zumino B, 1991. The twoparameter deformation of GL(2), its differential calculus, and Lie algebra. Zeitschrift für Physik C Particles and Fields, 49:317-

Yang C N, 1967. Some Exact Results for the Many-Body Problem in one Dimension with Repulsive 324. 